\title{
RELIC GRAVITATIONAL WAVES AND LIMITS ON INFLATION
}

\author{
L.P.Grishchuk \\ McDonnell Center for the Space Sciences, Physics Department \\ Washington University, St. Louis, MO 63130 \\ and \\ Sternberg Astronomical Institute, Moscow University \\ 119899 Moscow, V-234, Russia
}

\begin{abstract}
It is shown that only a narrow class of inflationary models can possibly agree with the available observational data on the anisotropy of the cosmic microwave background radiation (CMBR). These models may be governed by "matter" with the effective equation of state $-1.2<p / \epsilon<-0.6$ which includes the De-Sitter case $p / \epsilon=-1$.
\end{abstract}

PACS numbers: 98.80.Cq, 98.70.Vc, 04.30.+

Typeset using REVTEX 
The recent discovery of the angular variations in the CMBR [1] has strongly sharpened the issue of the origin and nature of the long-wavelength cosmological perturbations. The consequences of inflationary models are under active investigation (see, for instance, a recent paper [2] and references therein). It seems to me that only a narrow class of the inflationary models, discussed below, can possibly avoid theoretical and observational inconsistencies.

I am considering here relic gravitational waves. The variable gravitational field of all cosmological models (unless the cosmological scale factor $a(\eta)$ is such that $a^{\prime \prime}=0$ ), and inflationary models as a particular case, inevitably generate gravitational waves [3]. (The graviton creation in FRW fields was denied in the past and anisotropic models were claimed to be the only way to get a nonzero result, but now they are not considered anymore as a necesary condition.) The generating mechanism is quantum-mechanical in its nature, and the generated perturbations are always placed in squeezed vacuum quantum states (see [4] and references therein). This means that different modes of the created field are not totally independent, as is often assumed in the literature on inflation, but, on the contrary, some of them are highly correlated which leads to the picture of standing waves and modulated spectra. The generated gravitational waves inescapably produce the angular anisotropy in the CMBR. The angular correlation function for $\delta T / T$ variations caused by squeezed gravitational waves has been derived recently [4]. We will use it here in our analysis.

The dimensionless gravity-wave field, with all the normalization factors taken into account, can be written as

$$
h_{i j}(\eta, \mathbf{x})=4 \sqrt{\pi} \frac{l_{p l}}{a(\eta)} \frac{1}{(2 \pi)^{3 / 2}} \int_{-\infty}^{\infty} d^{3} \mathbf{n} \sum_{s=1}^{2} p_{i j}^{s}(\mathbf{n}) \frac{1}{\sqrt{2 n}}\left[c_{\mathbf{n}}^{s}(\eta) e^{i \mathbf{n x}}+c_{\mathbf{n}}^{s^{\dagger}}(\eta) e^{-i \mathbf{n x}}\right]
$$

where the scale factor $a(\eta), d s^{2}=a^{2}(\eta)\left(d \eta^{2}-d x^{2}-d y^{2}-d z^{2}\right)$, has the dimension of length and $l_{p l}=\left(G \hbar / c^{3}\right)^{1 / 2}$ is the Planck's length, all other quantities are dimensionless. The two "transverse-traceless" polarization tensors $p_{i j}^{s}(\mathbf{n})(s=1,2)$ satisfy the conditions $p_{i j}^{s}(\mathbf{n}) p^{s^{\prime} i j}(\mathbf{n})=2 \delta_{s s^{\prime}}, p_{i j}^{s}(-\mathbf{n})=p_{i j}^{s}(\mathbf{n})$. The time-dependent annihilation and creation operators $c_{\mathbf{n}}^{s}(\eta), c_{\mathbf{n}}^{s^{\dagger}}(\eta)$ can be written (for each $s$ ) as

$$
c_{\mathbf{n}}(\eta)=u_{n}(\eta) c_{\mathbf{n}}(0)+v_{n}(\eta) c_{-\mathbf{n}}^{\dagger}(0), \quad c_{\mathbf{n}}^{\dagger}(\eta)=u_{n}^{*}(\eta) c_{\mathbf{n}}^{\dagger}(0)+v_{n}^{*}(\eta) c_{-\mathbf{n}}(0),
$$


where $c_{\mathbf{n}}(0), c_{\mathbf{n}}^{\dagger}(0)$, are the initial values of the operators taken at some $\eta=\eta_{0}$ long before the interaction became effective. The complex functions $u_{n}(\eta), v_{n}(\eta)$ satisfy the equations

$$
i u_{n}^{\prime}=n u_{n}+i\left(a^{\prime} / a\right) v_{n}^{*}, \quad i v_{n}^{\prime}=n v_{n}+i\left(a^{\prime} / a\right) u_{n}^{*}
$$

where $^{\prime}=d / d \eta,\left|u_{n}\right|^{2}-\left|v_{n}\right|^{2}=1$ and $u_{n}(0)=1, v_{n}(0)=0$. It follows from these equations that the function $\mu_{n}(\eta) \equiv u_{n}(\eta)+v_{n}^{*}(\eta)$ obeys the equation $\mu_{n}^{\prime \prime}+\left(n^{2}-a^{\prime \prime} / a\right) \mu_{n}=0$ which is precisely the equation for classical complex $\mu$-amplitude [3] of the gravity-wave field.

The (Bogoliubov) transformation (2) can be written in a form involving the two-mode squeeze operator which demonstrates the inevitable appearance of squeezing in this kind of problems. In the Schrödinger picture, the initial vacuum state $|0\rangle, c_{\mathbf{n}}(0)|0\rangle=0$, evolves into a strongly squeezed vacuum state.

The angular correlation function for $\delta / T$ (see Eqs. (12), (13) in Ref. [4]) can be rearranged by using the "summation theorem" [5] and the formulas relating the Gegenbauer polynomials to the associated Legendre polynomials [6] and cast into an elegant exact form which directly involves the Legendre polynomials $P_{l}(\cos \delta)$ :

$$
\left\langle 0\left|\frac{\delta T}{T}\left(e_{1}^{k}\right) \frac{\delta T}{T}\left(e_{2}^{k}\right)\right| 0\right\rangle=l_{p l}^{2} \sum_{l=2}^{\infty} K_{l} P_{l}(\cos \delta)
$$

where $K_{l}=(2 l+1) l(l+1)[l(l+1)-2] F_{l}$,

$$
F_{l}=\int_{0}^{\infty} n^{2}\left|\int_{0}^{w_{1}} \frac{J_{l+\frac{1}{2}}(n w)}{(n w)^{5 / 2}} f_{n}\left(\eta_{R}-w\right) d w\right|^{2} d n
$$

and

$$
f_{n}\left(\eta_{R}-w\right)=\frac{1}{\sqrt{2 n}}\left(\mu_{n} / a\right)^{\prime} .
$$

The two unit vectors $e_{1}^{k}, e_{2}^{k}$ point out in the directions of observation and $\delta$ is the angle between them. The photons of CMBR are assumed to be emitted at $\eta=\eta_{E}$ and received at (present) time $\eta=\eta_{R} ; w=\eta_{R}-\eta, w_{1}=\eta_{R}-\eta_{E}$ (the Sachs-Wolfe effect [7]). Note that the correlation function, with no additional assumptions made whatsoever, is rotationally symmetric and its multipole expansion begins from $l=2$. 
The derived formula is universal and can be used with arbitrary $a(\eta)$. We will apply this formula to simple models consisting of three consecutive stages of expansion: inflationary ( $i$-stage), radiation-dominated ( $e$-stage) and matter-dominated ( $m$-stage) [8].

The scale factor of the model can be written at the three stages as follows. $i$-stage:

$$
a(\eta)=l_{0}|\eta|^{1+\beta}, \quad \eta \leq \eta_{1}, \quad \eta_{1}<0,
$$

e-stage:

$$
a(\eta)=l_{0} a_{e}\left(\eta-\eta_{e}\right), \quad \eta_{1} \leq \eta \leq \eta_{2}
$$

where $a_{e}=-(1+\beta)\left|\eta_{1}\right|^{\beta}, \eta_{e}=\frac{\beta}{1+\beta} \eta_{1}$,

$m$-stage:

$$
a(\eta)=l_{0} a_{m}\left(\eta-\eta_{m}\right)^{2}, \quad \eta_{2} \leq \eta,
$$

where $a_{m}=\left[a_{e} / 4\left(\eta_{2}-\eta_{e}\right)\right], \eta_{m}=-\eta_{2}+2 \eta_{e}$. The functions $a(\eta), a^{\prime}(\eta)$ are continuous at $\eta=\eta_{1}$ and $\eta=\eta_{2}$. All expanding models with $1+\beta<0$ ( $\eta$ must be negative if $1+\beta<0$ ) are inflationary in the sense that the length scale equal to the Hubble radius at some early time of expansion can grow at all three stages up to, at least, the size of the present day Hubble radius $l_{H}=a^{2} / a^{\prime}, \eta=\eta_{R}$. The case $\beta=-2$ corresponds to the De-Sitter expansion, the cases $\beta<-2$ correspond to the so called power-law inflation $\left(a(t) \sim t^{m}, m>1\right)$ and the cases $-2<\beta<-1$ (apparently, not having been analyzed before) correspond to the law of expansion $a(t) \sim|t|^{m}, m<-1, t<0$. The $i$-stage is governed by "matter" with the effective equation of state $p=q(\beta) \epsilon$, where $q(\beta)=(1-\beta) / 3(1+\beta)$ and $q(\beta)$ varies from $-1 / 3$ to $-\infty$ for $-\infty<\beta<-1$. Expansion is accompanied by the growth of energy density and curvature if $-2<\beta<-1$.

In realistic cosmological models $a\left(\eta_{E}\right) / a\left(\eta_{R}\right) \approx 10^{-3}, a\left(\eta_{2}\right) / a\left(\eta_{R}\right) \approx 10^{-4}$. Also, one has $3.10^{-32}<a\left(\eta_{1}\right) / a\left(\eta_{R}\right)<3.10^{-12}$ if one wants to commence the $e$-stage at densities not lower than the nuclear and not higher than the Planckian, or, in other words, if one wants 
the Hubble radius $l_{i}$ at the end of inflation, $l_{i}=-l_{o}(1+\beta)^{-1}\left|\eta_{1}\right|^{2+\beta}$, to be in the interval $1<l_{i} / l_{p l}<10^{40}$.

To define the numerical values of $\eta$ it is convenient to choose $\eta_{R}-\eta_{m}=1$. Then, $\left|\eta_{1}\right| \approx 5^{-2 / \beta}|1+\beta|^{-1 / \beta}\left(l_{o} / l_{H}\right)^{-1 / \beta}$ and $\left(l_{p l} / l_{o}\right)^{2} \approx\left(25 l_{p l} / l_{H}\right)^{2+\beta}|1+\beta|^{2(1+\beta)}\left(l_{i} / l_{p l}\right)^{\beta}$. The wavelength $\lambda=2 \pi a / n$ equal to $l_{H}$ has the wave number $n_{H}=4 \pi$, and the wavelength equal to the Hubble radius at $\eta=\eta_{2}$ corresponds to $n_{m} \approx 4 \pi \cdot 10^{2}$. The minimally sufficient inflation should begin not later than at $\eta_{b}=(1+\beta) / 2$, its variable gravitational field generates waves with wavelengths up to $l_{H}$. Inflation that started earlier inevitably generates the longer waves also.

The gravity-wave eguation using the scale factor of the form $a(\eta)=a_{o} \eta^{1+\beta}$ has been solved and the relation between the initial and final amplitudes has been derived earlier [3]. The waves start oscillating with the amplitude $B(n)$ which is related to the initial amplitude $A(n)$ by $B^{2}(n) \sim A^{2}(n)\left(n \eta_{1}\right)^{2(1+\beta)}$ (one can use formula (5b) from the second Ref. [3] in which the interpretation of the participating amplitudes should be reversed because, for $1+\beta<0$, the condition $(n \eta)^{2} \gg 1$ is satisfied initially and gets violated later on). Since the initial (vacuum) spectrum goes as $A(n) \sim n$ this leads to $B(n) \sim n^{\beta+2}$ and $B(n) \sim n^{o}$ for $\beta=-2$, that is, in case $\beta=-2$, all waves start oscillating with the same amplitude (the Harrison-Zeldovich spectrum). In case of $\beta<-2$ the spectrum gets "tilted" by increasing the relative contribution of longer waves, and in case of $\beta>-2$ - by increasing the relative contribution of shorter waves.

The exact solution to Eq. (3) for the complex function $\mu_{n}(\eta)$ satisfying the required initial data and continuous with its first time-derivative at the joining points $\eta_{1}$, $\eta_{2}$ has the following form.

$i$-stage:

$$
\mu_{n}(\eta)=(n \eta)^{1 / 2}\left[A_{1} J_{\beta+1 / 2}(n \eta)+A_{2} J_{-(\beta+1 / 2)}(n \eta)\right]
$$

(for a technical simplification we work solely with the Bessel functions and exclude the half-integer $\beta$ 's but the final result will be free of this limitation) where 


$$
A_{1}=-\frac{i}{\cos \beta \pi} \sqrt{\frac{\pi}{2}} e^{i\left(x_{0}+\pi \beta / 2\right)}, \quad A_{2}=i A_{1} e^{-i \pi \beta} \quad \text { and } \quad x_{o} \equiv n \eta_{o}, \quad n\left|\eta_{o}\right| \gg 2 \pi|1+\beta|
$$

e-stage:

$$
\mu_{n}(\eta)=B_{1} e^{-i n y}+B_{2} e^{i n y}
$$

where $y \equiv n\left(\eta-\eta_{e}\right)$,

$m$-stage:

$$
\begin{gathered}
\mu_{n}(\eta)=\sqrt{\frac{\pi z}{2}}\left(C_{1} J_{3 / 2}(z)+C_{2} J_{-3 / 2}(z)\right), \\
\left(\frac{\mu_{n}}{a}\right)^{\prime}=-\frac{n}{a} \sqrt{\frac{\pi z}{2}}\left(C_{1} J_{5 / 2}(z)-C_{2} J_{-5 / 2}(z)\right)
\end{gathered}
$$

where $z \equiv n\left(\eta-\eta_{m}\right)$. Note that $J_{-3 / 2}(z)$ represents the so-called decaying solution which is necessarily present.

The coefficients $B_{1}, B_{2}, C_{1}, C_{2}$, are determined by the continuos joining of the solutions. In particular,

$$
-C_{1}=B_{1}\left(\alpha_{2}+\beta_{2}^{*}\right)+B_{2}\left(\alpha_{2}^{*}+\beta_{2}\right), \quad-i C_{2}=B_{1}\left(\alpha_{2}-\beta_{2}^{*}\right)-B_{2}\left(\alpha_{2}^{*}-\beta_{2}\right)
$$

where $\alpha_{2}=e^{i y_{2}}\left(8 y_{2}^{2}-1+i 4 y_{2}\right) / 8 y_{2}^{2}, \beta_{2}=-e^{i 3 y_{2}} / 8 y_{2}^{2}, y_{2} \equiv n\left(\eta_{2}-\eta_{e}\right)$. We are only interested in modes that have interacted with the barrier $U(\eta)=a^{\prime \prime} / a$ and have been generated quantum-mechanically. Their wave numbers obey the condition $n\left|\eta_{1}\right| \ll 2 \pi|1+\beta|$ and for them

$$
B_{1} \approx-B_{2} \approx \frac{1}{2} e^{i x_{o}}(\beta+1) \psi(\beta)\left(n \eta_{1}\right)^{\beta} \equiv B
$$

where

$$
\psi(\beta) \equiv \sqrt{\frac{\pi}{2}} e^{i \pi \beta / 2}\left[\cos \beta \pi 2^{\beta+1 / 2} \Gamma(\beta+3 / 2)\right]^{-1}, \quad|\psi(\beta)|=1 \quad \text { for } \quad \beta=-2 .
$$

The values of $C_{1}, C_{2}$ depend on whether $y_{2} \gg 1$ or $y_{2} \ll 1$. For relatively short waves, $n \gg n_{m}$, one has approximately $C_{1} \approx-2 i B \sin y_{2}, C_{2} \approx 2 i B \cos y_{2}$. For longer waves, 
$n \ll n_{m}$, one has $C_{1} \approx-\frac{3 i}{2} B y_{2}^{-1}, C_{2} \approx-\frac{8 i}{45} B y_{2}^{4}, C_{2} \ll C_{1}$. The additional large factor $y_{2}^{-1}$ in $C_{1}$ reflects additional amplification of waves at $m$-stage.

For a qualitative description of amplitudes and spectral slopes we introduce "characteristic" spectral components of the field: $h(n)=l_{p l} n\left|\mu_{n}\right| / a$, and its first time-derivative: $h^{\prime}(n)=l_{p l}\left(\left|\mu_{n}\right| / a\right)^{\prime}$. We have $h(n) \sim \frac{l_{p l}}{a} n^{\beta+1} \sin \left[n\left(\eta-\eta_{e}\right)\right], h^{\prime}(n) \sim \frac{l_{p l}}{a} n^{\beta+1} \cos \left[n\left(\eta-\eta_{e}\right)\right]$ for $n \gg n_{m}$, and $h(n) \sim \frac{l_{p l}}{a} n^{\beta} \cos \left[n\left(\eta-\eta_{m}\right)\right], h^{\prime}(n) \sim \frac{l_{p l}}{a} n^{\beta} \sin \left[n\left(\eta-\eta_{m}\right)\right]$ for $n_{H} \gg n \gg n_{m}$. For $n \ll n_{H}$ one can use the approximation $z \ll 1$ in Eqs. (6), (7) and obtain $h(n) \approx \frac{l_{p l}}{l_{o}}|\psi(\beta)| n^{\beta+2}, h^{\prime}(n) \approx \frac{l_{p l}}{5 l_{o}}|\psi(\beta)| z n^{\beta+2}$.

We will now start deriving restrictions on inflationary models. Astrophysically interesting and consistent values of $h$ require $h\left(n_{H}\right)$ to be not much larger or much smaller than $10^{-4}$. The mean square value of the field diverges in the limit of small $n$ (i.e. wavelengths much longer than $l_{H}$ ) for all $\beta \leq-2:\left\langle h^{2}\right\rangle \approx\left(l_{p l} / l_{o}\right)^{2}|\psi(\beta)|^{2} \int \frac{d n}{n} n^{\beta+2}$. This does not allow the duration of inflation to be arbitrarily long. In case of $\beta=-2$ the divergence is logarithmic and the restriction on duration is very mild but it becomes increasingly severer for $\beta<-2$. For $\beta \approx-7$, even the minimally sufficient duration of inflation does not help as the $h\left(n_{H}\right)$ strongly exceeds $10^{-4}$ even if the largest allowed $l_{i}, l_{i} \approx 10^{40} l_{p l}$, is chosen. On the other hand, for $\beta>-2$ there is no problem with the long-wavelength divergence but the amplitudes very quickly become too small. For $\beta \approx-1.8$, the $h\left(n_{H}\right)$ becomes smaller than the required level even if the smallest allowed $l_{i}, l_{i} \approx l_{p l}$, is chosen. (One may argue that the values $l_{i} \ll l_{p l}$ do not necesserily invalidate the analysis and can also be allowed, although they imply the over-Planckian densities, since the wavelengths of our interest are much longer than $l_{p l}$ all the way up from the beginning of inflation. However, we will confine in this paper to the requirement $l_{i}>l_{p l}$ which leads to $\beta<-1.8$.)

Additional restrictions come from the $\delta T / T$ considerations. We will be interested in the lower multipoles $l, l \leq 30$, to which the waves $n \gg n_{m}$ give a negligibly small contribution. Neglecting the second term in Eq. (7) one can write Eq. (5) as

$$
F_{l} \approx \frac{9 \pi}{4 l_{o}^{2}}|\psi(\beta)|^{2} \int_{0}^{n_{m}} n^{2 \beta+3}\left|\int_{o}^{n w_{1}} \frac{J_{l+1 / 2}(x)}{x^{5 / 2}} \frac{J_{5 / 2}(n-x)}{(n-x)^{3 / 2}} d x\right|^{2} d n
$$


where $w_{1} \approx 1-3 \cdot 10^{-2}$. In the limit of long waves one can use the small argument approximation for the Bessel functions and write

$$
F_{l} \approx \frac{9 \pi}{4 l_{o}^{2}}|\psi(\beta)|^{2} \phi^{2}(l) \int n^{2 \beta+2 l+3} d n,
$$

where $\phi(l)=\left[15 \sqrt{\pi} 2^{l} \Gamma(l+1 / 2) l(l-1)\right]^{-1}$. The quadrupole component $K_{2}$ is divergent in the limit of small $n$ for all $\beta \leq-4$ so these values of $\beta$ should be excluded unless the duration of inflation is precisely tuned. (Interestingly enough, the minimally sufficient inflation may be a likely outcome of certain quantum-cosmological models [9].)

The multipole distributions $K_{l}$ for some models have been numerically computed by A. Wiseman. For each $l$, the main contribution to $F_{l}$ comes, as one might expect, from the interval of $n$ between $n \approx l$ and $n \approx 2 l$. In particular, $K_{2}$ is dominated by waves longer than $l_{H}[10]$. The absolute values of $K_{l}$ depend on $\beta$ and $l_{o}$. For instance, the quadrupole component $10^{2}\left(l_{o} / l_{p l}\right)^{2} K_{2}$ has the following numerical values: $9.7,7.3,5.6,7.4$, if one takes $\beta=-1.8,-2.0,-2.4,-3.0$, correspondingly. The multipole distributions normalized to $K_{2}$, that is the functions $K_{l} / K_{2}$, are independent of $l_{o}$. They are shown in Fig. 1. For completeness we have included also the extreme cases $\beta=-1.6,-1.1$, though they would imply strongly over-Planckian densities at the end of inflation, as was explained above. It is worth noting that the quadrupole component exceeds other multipoles and $K_{3} / K_{2}<0.6$ for all models with $\beta<-1.8$. The results for the $\beta=-2$ case are in qualitative agreement with those in Ref. 11. It remains to be seen which of these distributions can survive after comparison with the detailed COBE-type observations. One should bear in mind, of course, that predictions for $K_{l}$ are statistical and should be augmented with variancies based on the statistics of squeezed quantum states.

In conclusion, the inflationary models governed by "matter" with the effective equation of state $-1.2<p / \epsilon<-0.6$ seemingly avoid theoretical difficulties and some of them can possibly withstand comparison with the observations. (The models include the De-Sitter case if the duration of expansion was not excessively long.) Taking into account the density perturbations can only make this interval narrower. 
I would like to thank Alan Wiseman for his help.

This work was supported in part by NASA grant NAGW 2902 and NSF grant 89-22140. 


\section{REFERENCES}

[1] G. Smoot et al., Astrophys. J. (to be published); N. Wright et al., ibid. (to be published); CMB Workshop 1992, Berkeley, December 1992; Texas/PASCOS '92 meeting, Berkeley, December 1992 (to be published).

[2] R. L. Davis, H. M. Hodges, G. F. Smoot, P. J. Steinhardt and M. S. Turner, Phys. Rev. Lett. 69, 1856 (1992).

[3] L. P. Grishchuk, Zh. Eks. Teor. Fiz. 67, 825 (1974) [Sov. Phys. JETP 40, 409 (1975)]; Ann. N.Y. Acad. Sci. 302, 439 (1977).

[4] L. P. Grishchuk, Phys. Rev. Lett. (19 April 1993, to appear)

[5] I. S. Gradshteyn and I. M. Ryzhik, Table of Integrals, Series, and Products (Academic Press, 1980), formula 8.532.

[6] Ph. M. Morse and H. Feshbach, Methods of Theoretical Physics, (McGraw-Hill, 1953), p. 728.

[7] R. K. Sachs and A. M. Wolfe, Astrophys. J. 147, 73 (1967).

[8] L. P. Grishchuk and Ya. B. Zeldovich, in: Quantum Structure of Space and Time, eds. M. Duff and C. Isham, (Cambridge University Press, 1982), p. 409.

[9] L. P. Grishchuk, Phys. Rev. D45, 4717 (1992); M. S. Turner, Phys. Rev. D44, 3737 (1991).

[10] L. P. Grishchuk and Ya. B. Zeldovich, Astron. Zh. 55, 209 (1978) [Sov. Astron. 22125 (1978)].

[11] R. Fabbri and M. D. Pollock, Phys. Lett. 125B 445 (1983); V. A. Rubakov, M. V. Sazhin and A. V. Veryaskin, Phys. Lett. 115B, 189 (1982); L. F. Abbott and M. B. Wise, Nucl. Phys. B244, 541 (1984); A. A. Starobinsky, Sov. Astron. Lett. 11, 133 (1985); M. White, Phys. Rev. D46, 4198 (1992). 


\section{FIGURES}

FIG. 1. The normalized multipole distributions. 